Editorial: Open Access

\title{
Bring New Advances of Basic Medical Research Closer to Patient Care
}

\section{Xiaoming Yang}

\author{
Department of Radiology, University of Washington School of Medicine, Seattle, Washington, USA
}

*Corresponding author: Xiaoming Yang, Professor of Radiology, Director, Image-Guided Bio-Molecular Interventions Research, Department of Radiology, University of Washington School of Medicine, Seattle, Washington, USA, Tel: 206-685-6967; Fax: 206-543-3495; E-mail: xmyang@u.washington.edu

With great pleasure, I welcome you to the inaugural issue of the International Archives of Translational Medicine.

The rapid progress in basic medical research is constantly reframing the paradigm how we prevent, diagnose and treat human diseases. Scientists from various disciplines around the world have performed outstanding works in developing and establishing innovative methods and technologies for screening different diseases, such as cancer and atherosclerotic cardiovascular disease, as well as early diagnosis and effective treatment of such life-threatening illnesses. The tremendous effort by basic scientists have covered wide spectrum of research fields, from genetics to molecular diagnostics, nanotechnology to targeted tracers/therapeutics, bio-engineering to bio-informatics, and minimally-invasive instruments to diagnostic/ therapeutic equipment. However, clinical applications of these medical advances have been limited. This limit could at least partially be attributed to a gap existed between basic science and clinical medicine, where the basic scientists have no direct access to patient care while clinicians are occupied by daily clinical responsibilities and lack the time and convenient channel to learn new discoveries in basic medical science. In order to bring the two parties together, a "bridge" needs to be built between basic science and clinical practice, so called "translational medicine" that has been endorsed by the US National Institute of Health [1].

The aim of opening this journal, International Archives of Translational Medicine, is to facilitate such medical translation from laboratory bench to preclinical animal studies, and ultimately to clinical practice, i.e., building the bridge. We hope that this journal will provoke common interests, brainstorm new ideas, encourage collaborations among professionals in both basic science and clinical medicine, and bring innovative medical concepts and technologies one step closer to patience care.

\section{References}

1. News (2010) NIH director wins bid for translational medicine centre: New nexus will bridge basic and clinical research to improve health. Nature. online 8 December
Citation: Yang X (2015) Bring New Advances of Basic Medical Research Closer to Patient Care. Int Arch Transl Med 1:001e

Received: February 23, 2015: Accepted: February 24, 2015: Published: February 25, 2015 the Creative Commons Attribution License, which permits unrestricted use, distribution, and reproduction in any medium, provided the original author and source are credited. 